\title{
Hidden metal-insulator transition in manganites synthesized via a controllable oxidation
}

\author{
Chuangye Song 1 , Iftikhar Ahmed Malik ${ }^{1}$, Menglei Li $^{2}$, Qinghua Zhang ${ }^{3}$, Lichen Wang ${ }^{2}$, \\ Jing Wang ${ }^{1,4}$, Rongyan Chen ${ }^{1,5}$, Renkui Zheng ${ }^{6}$, Shuai Dong ${ }^{7}$, Lin $\mathrm{Gu}^{3}$, Wenhui Duan ${ }^{8}$, \\ Ce-Wen $\mathrm{Nan}^{4}$ and Jinxing Zhang ${ }^{1^{*}}$
}

\begin{abstract}
Oxygen usually plays crucial roles in tuning the phase structures and functionalities of complex oxides such as high temperature superconductivity, colossal magnetoresistance, catalysis, etc. Effective and considerable control of the oxygen content in those functional oxides could be highly desired. Here, using perovskite manganite $\left(\mathrm{La}_{0.5} \mathrm{Sr}_{0.5}\right) \mathrm{MnO}_{3}$ as a paradigm, we develop a new pathway to synthesize the epitaxial thin films assisted by an in-situ chemical process, where the oxygen content can be precisely controlled by varying oxidative activity tuned by the atmospheric temperature $\left(T_{\text {atm }}\right)$ during the growth. A hidden metal-insulator transition (MIT) emerges due to the phase competition, which is never shown in the phase diagram of this classic manganite. The oxygenmediated interaction between $\mathrm{Mn}$ ions together with the change of carrier density might be responsible for this emerging phase, which is compatible with the results of firstprinciple calculations. This work demonstrates that, apart from traditional cation doping, a precise modulation of anion $\left(\mathrm{O}^{2-}, \mathrm{S}^{2-}\right.$, etc.) may provide a new strategy to control phase structures and functionalities of epitaxial compound thin films.
\end{abstract}

Keywords: oxidative-activity, oxygen-content, manganites, hidden metal-insulator transition

\section{INTRODUCTION}

As a prominent control parameter in oxide compounds, oxygen plays a crucial role in strongly correlated beha- viors and functionalities, which is usually coupled with other degrees of freedom such as spin, orbital, charge and lattice $[1,2]$. Excess oxygen $\left(\mathrm{E}_{\mathrm{O}}\right)$ can induce secondary oxidized phases in perovskite manganites such as $\mathrm{LaMnO}_{3+\delta}$ and $\mathrm{YMnO}_{3+\delta}$ [3]. Oxygen vacancy $\left(\mathrm{V}_{\mathrm{O}}\right)$ can be the source of mobile carriers as an electron donor [4] in high temperature superconductors [1], solid-oxide fuel cells [5], gas sensors and multiferroics [6], etc. The existence of $\mathrm{V}_{\mathrm{O}}$ and/or $\mathrm{E}_{\mathrm{O}}$ at the surface [7], interface [8], as well as their bulk forms [9] of various oxide materials can also give rise to intriguing insights for both the fundamental physics/chemistry and potential opportunities of practical applications. In order to achieve the integration of oxide-based electronic devices, there is a strong impetus to pursue a precise and homogeneous control of oxygen component during the growth of high-quality oxide thin films. Advanced epitaxial growth techniques (e.g., pulsed laser deposition (PLD), laser-molecular beam epitaxy (Laser-MBE), sputtering deposition, etc.) are employed to in-situ tune oxygen-stoichiometry under different oxygen atmospheres. From the analysis of electron energy-loss spectra, plenty of cation and oxygen vacancies may appear close to the surface, boundary and interface of epitaxial films due to the deficient inactive oxygen ("cold oxygen") during the growth [10-12], leading to severe deterioration of their structures and properties of some epitaxial oxide films. Ex-situ annealing under a flowing active oxygen ("hot oxygen") atmosphere

\footnotetext{
${ }^{1}$ Department of physics, Beijing Normal University, Beijing 100875, China

${ }^{2}$ Department of Physics, Capital Normal University, Beijing 100048, China

${ }^{3}$ Institute of physics, Chinese Academy of Sciences, Beijing 100190, China

${ }^{4}$ School of Materials Science and Engineering, Tsinghua University, Beijing 100084, China

${ }^{5}$ Center for Advanced Quantum Studies, Beijing Normal University, Beijing 100875, China

${ }^{6}$ State Key Laboratory of High Performance Ceramics and Superfine Microstructure, Shanghai Institute of Ceramics, Chinese Academy of Sciences, Shanghai 200050, China

${ }^{7}$ School of Physics, Southeast University, Nanjing 211189, China

${ }^{8}$ Department of Physics and State Key Laboratory of Low-Dimensional Quantum Physics, Tsinghua University, Beijing 100084, China

* Corresponding author (email: jxzhang@bnu.edu.cn)
} 
is also a popular way to reduce the oxygen vacancies and defects. However, desired homogeneous control of oxygen content and stoichiometry possibly occurs at only top layers of some films [13], indicating an oxygen distribution along the thickness direction of the films, especially for the compounds with a relative short oxygen diffusion length.

Under these circumstances, there is a strong motivation to explore a new film growth strategy, where both the precise control and uniform distribution of oxygen content may be achieved, especially for those dysoxidizable materials. In conventional physical deposition procedures, such as classic PLD [14], the expansion of plasma plume, corresponding to the composition and mean energy distributions of desorbed particles, is very sensitive to the chemical atmospheres $[15,16]$ and can determine the growth dynamics/compositions. When they reach the subsequent substrates, ionic and excited species suffer a prominent delay of kinetic energy transfer in the relatively inert ("cold") conditions [17], accompanied with a decrease of the sufficient reaction and crystallization $[18,19]$. This is one of the major factors that may induce off-stoichiometry and defects in oxide thin films [10]. Contrary to the physical depositions, chemical vapor deposition (CVD) provides a more chemically-reactive atmosphere during the growth of oxide nanocrystals and thin films [20], so that the excited oxygen molecule and oxygen-metal species may endure a low decay rate and a significant enhanced reactivity when they reach the substrates. Nevertheless, the relatively low thermal excitation energy (lower than the vapor point of most transition metal oxides) and poor stoichiometric control of metal elements restrict the effective high-quality growth of correlated complex oxides. Therefore, a laser-ablated process in CVD system may provide a new strategy to control epitaxial complex oxide growth with a precise and homogeneous elemental stoichiometry. Among the functional complex oxides, perovskite manganites possess intriguing structures and strong electron correlations, exhibiting high spin-polarization, high Curie temperature in oxide magnets and colossal magnetoresistance (CMR) $[21,22] . \mathrm{La}_{0.5} \mathrm{Sr}_{0.5} \mathrm{MnO}_{3}$ (LSMO) is a classic manganite in CMR family, which lies in the boundary between ferromagnetic/antiferromagnetic metallic phases modulated by the 50/50 ratio of cation $\mathrm{La}^{3+}$ and $\mathrm{Sr}^{2+}$ (half-doped) [23-25]. It has attracted peoples' interest to investigate its intriguing properties under various stimuli (e.g., epitaxial strain [26-29], electric [30] and magnetic field [31]). None of these methods succeeded in creating a charge ordering phase and corresponding CMR behavior [27], which is usually observed and controlled in other manganites with an A-site elemental ratio about 50/50 [32].

In this work, a laser-ablated CVD is employed in LSMO to demonstrate an in-situ precise control of oxygen content with homogenous distribution during highquality epitaxial growth. A hidden antiferromagnetic/insulating-to-ferromagnetic/metal transition is discovered around $150 \mathrm{~K}$ in this LSMO with optimized oxygen content, which is not shown in its phase diagram. This antiferromagnetic/insulating phase is very sensitive to the oxygen content $\left(\mathrm{E}_{\mathrm{O}}\right.$ or $\left.\mathrm{V}_{\mathrm{O}}\right)$ that can be tuned by varying the atmospheric temperature $\left(T_{\text {atm }}\right)$. The ferromagnetic (FM) and antiferromagnetic (AFM) phase separation/ transition was directly visualized using magnetic force microscopy (MFM) at $4 \mathrm{~K}$ as a function of magnetic field. The detailed understanding of correlation between electronic/magnetic phases and chemical structures of these LSMO films with a precise control of oxygen helps unveil a temperature-oxygen stoichiometry $(\delta)$ phase diagram.

\section{RESULTS AND DISCUSSION}

Fig. 1a shows the schematic design of our laser-ablated CVD equipped with a quartz tube. Ceramic target and substrate are placed at the center of the tube. The branch tube is tilted by $45^{\circ}$. Laser beam (purple color) goes through the branch tube and interacts with the ceramic target to generate plasma plume (light blue). The flowing high-temperature oxygen (orange cluster) with high chemical-reactivity recombines layer by layer with the cations and composites on the substrate (red rectangle). The $T_{\text {atm }}$ of the laser-ablated CVD system can be maintained uniform up to $1,500 \mathrm{~K}$ for the entire space inside the tube. Two thermocouples are placed near the substrate and at the center of the tube respectively, which reveals that temperature of the substrate has the same value as the $T_{\mathrm{atm}}$. Using this new method, epitaxial LSMO thin films have been grown on perovskite (001)-oriented $\mathrm{LaAlO}_{3}(\mathrm{LAO})$. X-ray diffraction (XRD) pattern of LSMO film on LAO substrate (Fig. $1 \mathrm{~b}$ and Fig. S1a, see Supplementary information) reveals a high-quality epitaxial structure with a set of (001)-oriented diffraction peaks. Energy dispersive X-ray spectroscopy analysis (Fig. S1b) confirms that the chemical ratio of the as-grown LSMO epitaxial film is well consistent with the perovskite stoichiometry. Crystal structure of the LSMO thin films is characterized by high-resolution transmission electron microscopy (TEM). The high-angle annular dark-field (HAADF) micrograph of the LSMO film on LAO taken by an aberration-corrected STEM is shown in Fig. 1c. Since the intensity of an atomic column is approximately 
a

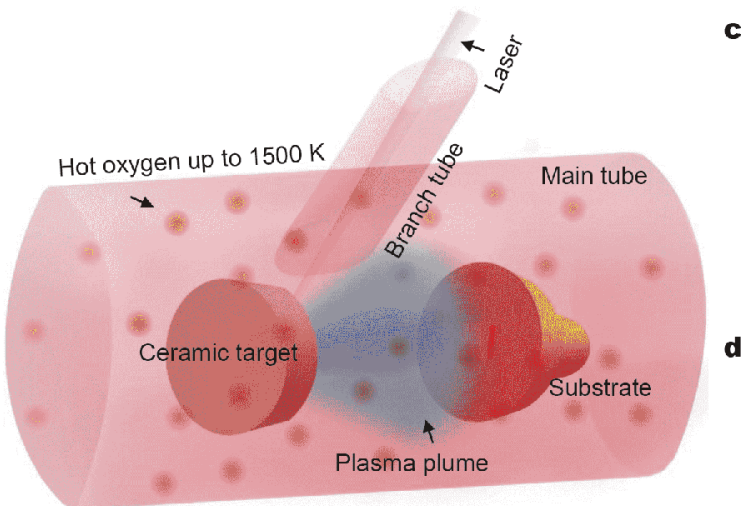

b

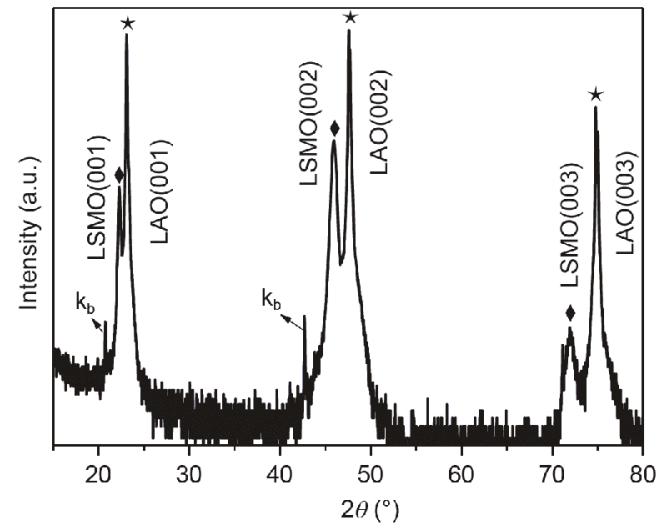

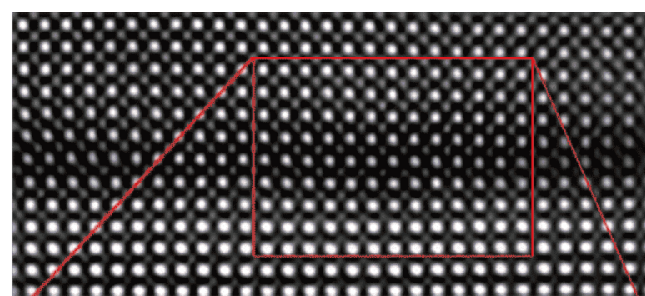

d
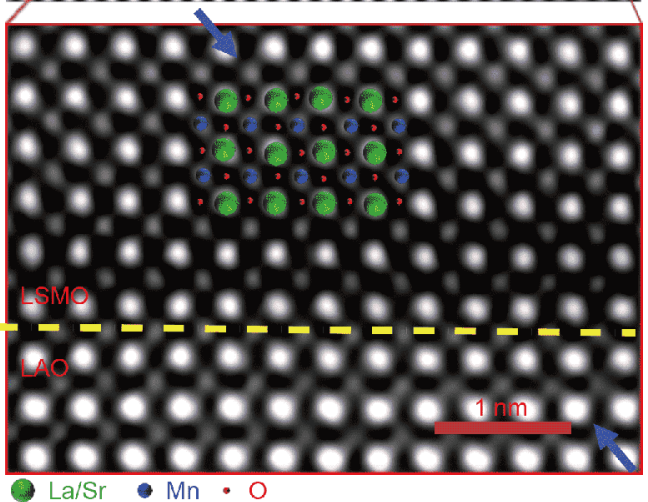

$\mathbf{e}$

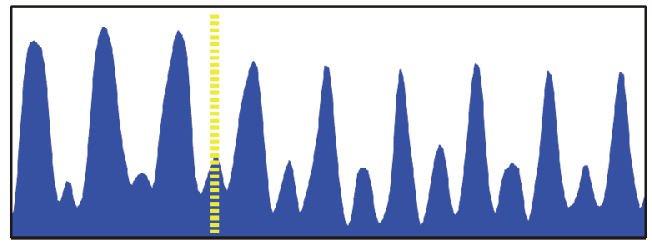

Figure 1 High-quality crystallographic structure of the epitaxial LSMO films by laser-ablated CVD. (a) Schematic of our home-designed laser-ablated CVD. (b) X-ray diffraction pattern of $120 \mathrm{~nm}$ LSMO thin film grown on LAO substrate, indicating a typical (001)-oriented epitaxial oxide thin film. (c) HAADF micrograph of the LSMO film on LAO substrate taken by an aberration-corrected STEM. (d) Zoom-in image of the rectangular solid area in (c). The yellow-dashed line indicates the LSMO/LAO interface location. (e) Intensity line profile of the region marked by the purple arrows in (d).

proportional to $Z^{1.7}$ ( $Z$, atomic number), the brighter area in Fig. $1 \mathrm{c}$ corresponds to the LAO substrate, and the less bright area to the LSMO film. Zoom-in image of the rectangular solid area in Fig. 1c is presented in Fig. 1d, and the intensity line profiles along the purple arrow (Fig. 1d) arranging in a way to cross both the A-site cations (i.e., $\mathrm{La} / \mathrm{Sr}$ ) and the $\mathrm{B}$-site cations (i.e., $\mathrm{Mn} / \mathrm{Al}$ ) is shown in Fig. 1e. An abrupt contrast of $\mathrm{La}$ and $\mathrm{La} / \mathrm{Sr}$ provides a well-defined localization of the interface, which is marked by the yellow dashed line in Fig. 1e. The epitaxial growth of the high-quality LSMO film with this laser-ablated CVD provides us a platform to explore the competing electronic/magnetic phases by in-situ tuning chemical reactivity of "hot oxygen".

Three representative LSMO films (approximately $120 \mathrm{~nm}$ ) were obtained by laser-ablated CVD with $T_{\text {atm }}$ at $973,1,073$ and $1,173 \mathrm{~K}$ respectively. The laser $(248 \mathrm{~nm}$ excimer) energy density was about $2 \mathrm{~J} \mathrm{~cm}^{-2}$ with the repetition rate of $3 \mathrm{~Hz}$ in the oxygen environment of $20 \mathrm{~Pa}$ during the growth. The (002)-peak in Fig. 2a gradually shifts towards lower angle as $T_{\text {atm }}$ decreases from 1,173 K to $973 \mathrm{~K}$, which indicates an expansion of $c$-axis lattice constant from $3.863 \AA$ to $3.899 \AA$, a value larger than the bulk value of $3.858 \AA$. The expansion of the lattice constant is due to the effect of oxygen vacancies that weaken the lattice interatomic forces [33]. Considering that different $\mathrm{Mn}$ valence states have different ionic radii $\left(\mathrm{Mn}^{3+}\right.$ $=0.645 \AA \mathrm{Mn}^{4+}=0.53 \AA$ ), the existence of more $\mathrm{Mn}^{3+}$ is most likely responsible for the expansion of the lattice constant with lower $T_{\text {atm }}$, other than $\mathrm{Mn}^{4+}$ ions which are usually caused by excess oxygen [34]. The Mn 3s has a relatively higher sensitivity for determining $\mathrm{Mn}$ valence states than Mn 2p signal due to the strong influence from the outer electron configuration. The value of $\mathrm{Mn} 3 \mathrm{~s}$ exchange splitting $\Delta E_{3 \mathrm{~s}}$ has a linear dependence on the $\mathrm{Mn}$ valence states, $V_{\mathrm{Mn}}=9.67-1.27 \Delta E_{3 \mathrm{~s}} / \mathrm{eV}$ [11]. A decrease of the Mn 3s exchange splitting energy in Fig. 2b is qualitatively visible with increasing $T_{\text {atm }}$, which implies the increasing of $\mathrm{Mn}$ valence. Fig. 2c shows the peak splitting of Mn $2 \mathrm{p}$ in the three films mentioned above. It 

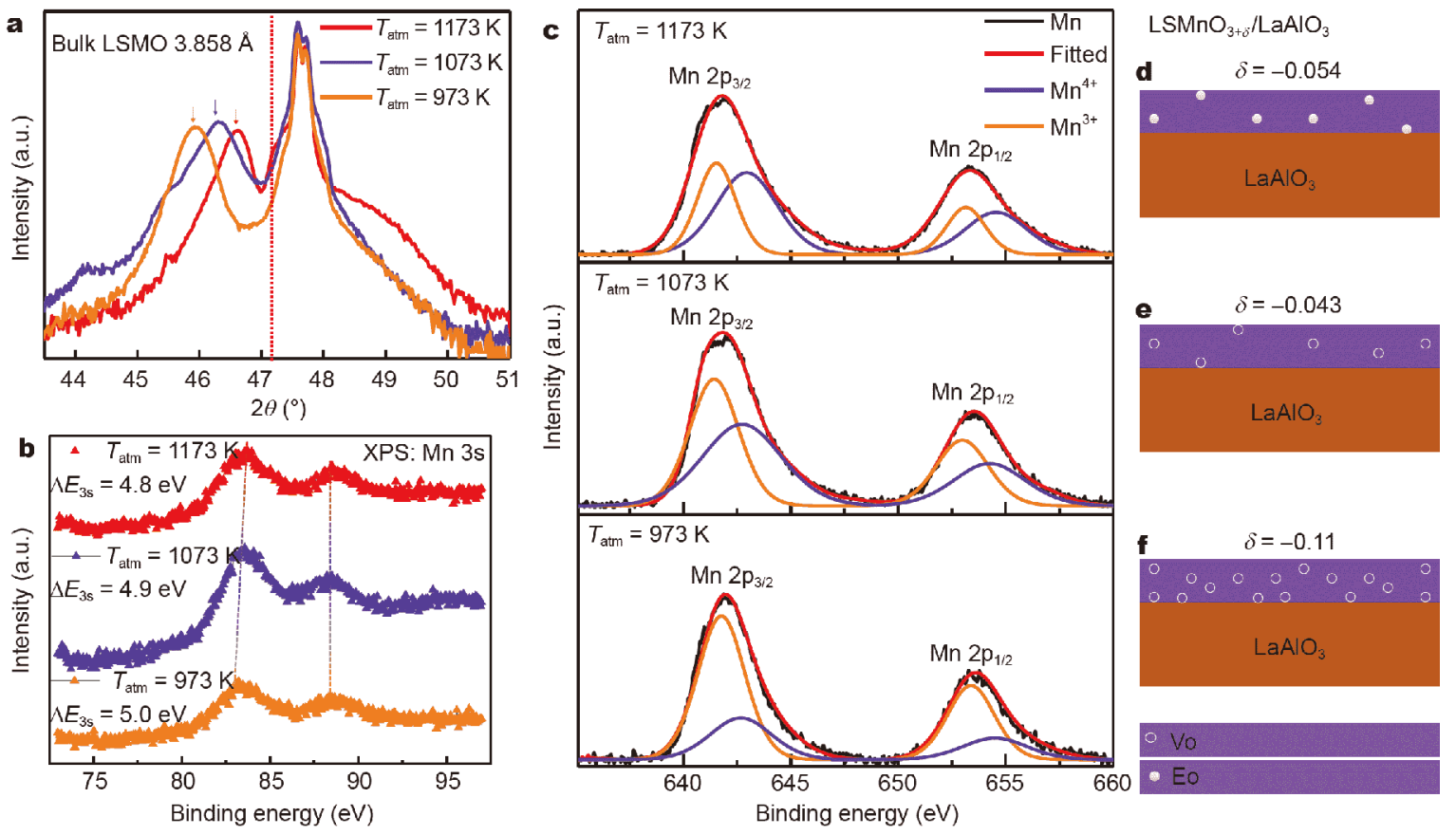

Figure $2 T_{\text {atm}}$-dependent lattice and valence structure. (a) An expanded view of the (002) peak of LSMO films acquired at $T_{\text {atm }}=1,173,1,073$ and $973 \mathrm{~K}$. (b) Mn 3s XPS spectra of the LSMO films. (c) The XPS peaks splitting analysis of Mn 2p of the three LSMO films after subtraction of the background. (d-f) Schematics of the nonstoichiometric oxygen content $(\delta)$ distribution in LSMO films.

gives the change of $\mathrm{Mn}^{3+} / \mathrm{Mn}^{4+}$ ratio estimated by the areas of the $\mathrm{Mn}^{3+}$ and $\mathrm{Mn}^{4+}$ peaks, which permits us, using the principle of electroneutrality, to approximately calculate the nonstoichiometric oxygen content $\delta$ from $(0.5-2 \delta) /(0.5+2 \delta)$, where $\delta$ denotes the nonstoichiometric oxygen content of $\mathrm{LSMO}_{3+\delta}$. Fig. $2 \mathrm{~d}-\mathrm{f}$ show schematically the corresponding $\delta$ varying from -0.11 to 0.054 as $T_{\text {atm }}$ increases from $973 \mathrm{~K}$ to $1,173 \mathrm{~K}$, which is well consistent with the valence change derived from the Mn 3s exchange splitting energy in Fig. 2b. Depth-dependent XPS measurements on films grown by the laser-ablated CVD system are also performed (Fig. S2), which reveals a homogenous distribution of manganese valence states. Thus, oxygen stoichiometry is efficiently tuned from a $V_{O}$ to $\mathrm{E}_{\mathrm{O}}$ state. It implies that "hot oxygen" in the laserablated CVD system has a remarkable influence on the chemical-reactivity of oxygen-related species and chemical sates of $\mathrm{Mn}$ in the film. Both $\mathrm{V}_{\mathrm{O}}$ and $\mathrm{E}_{\mathrm{O}}$ can be considered as mobile carrier donors, which will in turn affect the carrier density and mobility. Moreover, the magnetic and electric transport behaviors have a close relationship with the carrier itinerant activity. As shown in Fig. 3a, as $T_{\text {atm }}$ changes from 973 to $1,173 \mathrm{~K}$, insulating phase changes to metallic phase which corresponds to the change of $\delta$ from -0.11 to 0.054 . In comparison with laser-ablated CVD growth, traditional PLD is also used to grow LSMO films on LAO at various substrate temperatures, which shows insulating behavior and off-stoichiometry even after post annealing (Figs S3, S4) as also observed elsewhere [26,27].

Temperature- and magnetic-field-dependent magnetizations of these films by laser-ablated CVD are given in Fig. 3b. Curie temperature and saturation magnetization monotonically increase with increasing $T_{\text {atm. }}$. For the film grown at $T_{\text {atm }}=1,073 \mathrm{~K}$, zero-field cooling measurement reveals an obvious suppression of magnetization below $150 \mathrm{~K}$ (the magnetization at $4 \mathrm{~K}$ is about $2.9 \mu_{\mathrm{B}} / \mathrm{Mn}$, which is smaller than previous experimental and theoretical studies $\left(3.28 \mu_{\mathrm{B}} / \mathrm{Mn}\right)$ [35]), corresponding to a ground AFM/FM phase competition/separation and a hidden MIT. This hidden MIT is so sensitive to the oxygen content and can be controlled by a precise oxidization. External magnetic field (up to $5 \mathrm{~T}$ ) applied both in the plane and out of the plane (Fig. 3c) can partially suppress the AFM-insulating phase and reveals a magnetoresistance (MR) up to $70 \%$ at $4 \mathrm{~K}$.

To further elucidate the underlying physical mechanism of the phase transition at low temperature, MFM is used to visualize FM and AFM phase at mesoscopic scale. Due to its sensitivity to stray field gradients and having 


\section{a}

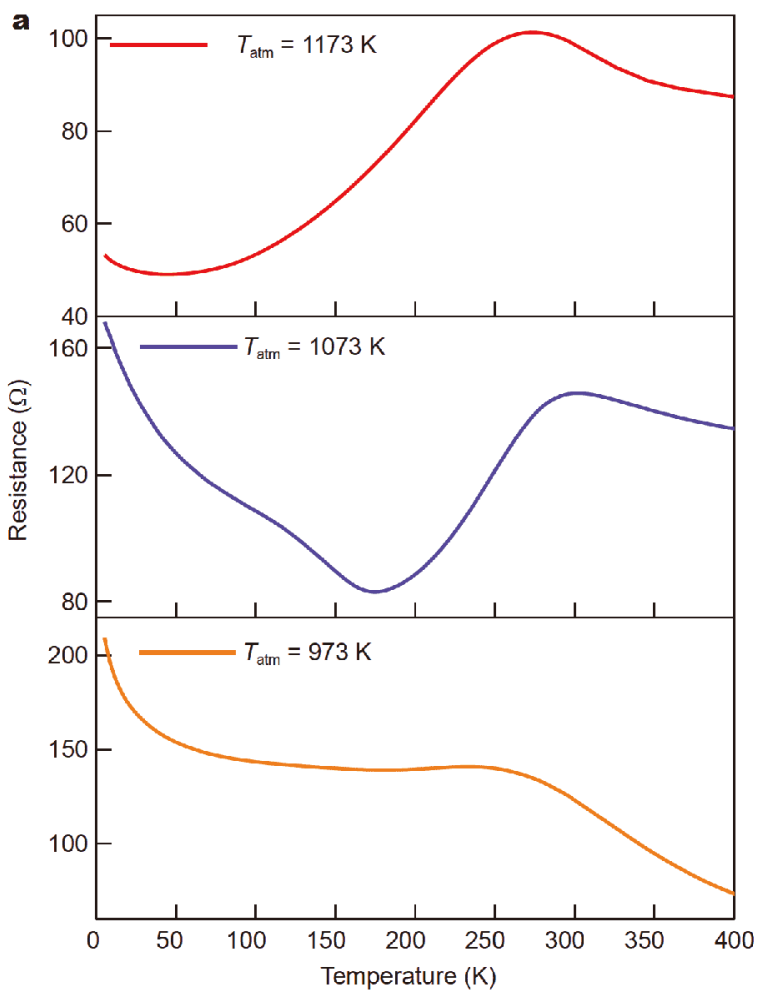

b

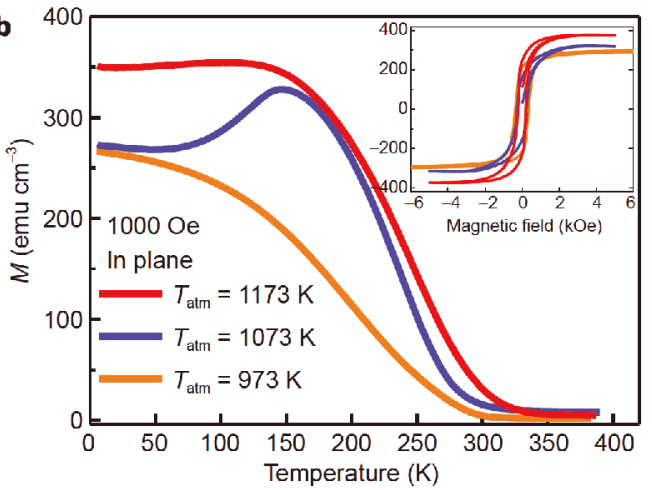

c

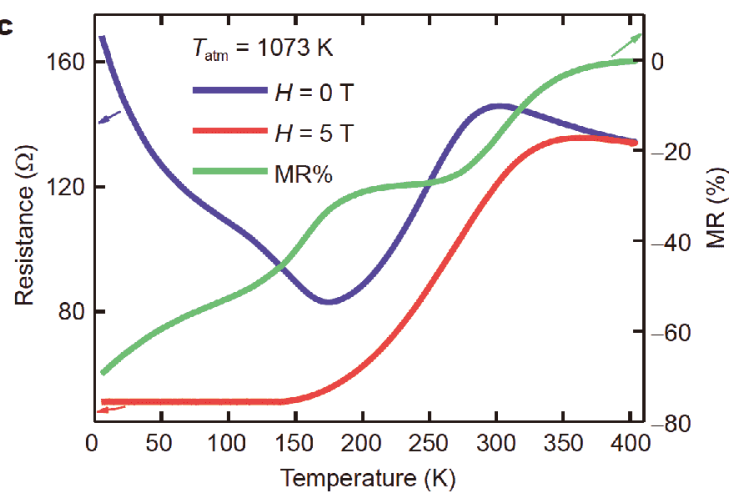

Figure 3 Hidden MIT and corresponding CMR. (a) Temperature-dependent resistance of films grown at varying $T_{\text {atm }}(973,1,073$ and 1,173 K). (b) Temperature-dependent magnetization measured during warming under 1,000 Oe after zero field cooling. Inset in (b) shows magnetic-fielddependent magnetization at $4 \mathrm{~K}$ after zero field cooling. (c) Temperature-dependent magnetoresistance (MR) of LSMO/LAO film grown at $T_{\text {atm }}=$ $1,073 \mathrm{~K}$ by laser-ablated CVD system.
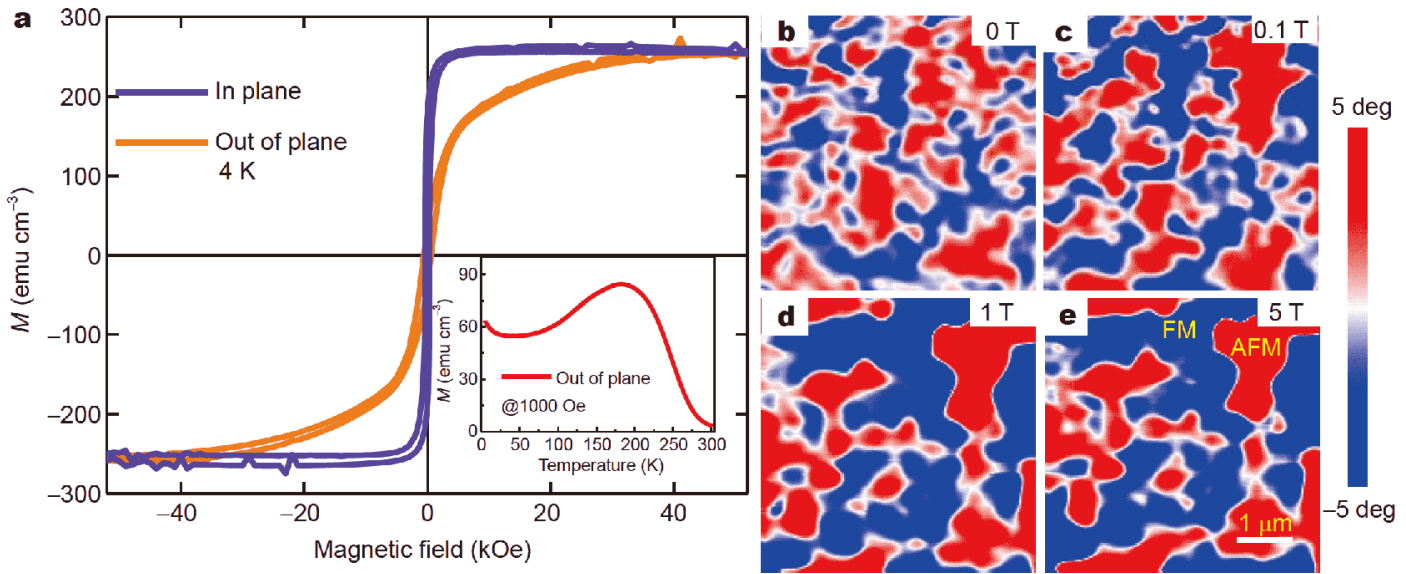

Figure 4 Direct visualization of phase separation using MFM at 4 K. (a) Magnetization as a function of magnetic field along in plane and out of plane of film grown at $T_{\text {atm }}=1,073 \mathrm{~K}$. Inset in (a) is the temperature-dependent magnetization measured during warming under 1,000 Oe after zero field cooling. (b-e) Magnetic-field-dependent MFM for direct visualization of FM and AFM regions. External magnetic field is applied perpendicular to the film surface in the same direction of initial magnetization of tip.

no response to the paramagnetic (PM) and AFM phase, MFM has been used to direct visualize FM and AFM phases in mixed-phase magnets [36-38]. Fig. 4b-e show field-dependent MFM images of LSMO thin film $\left(T_{\mathrm{atm}}=\right.$ 

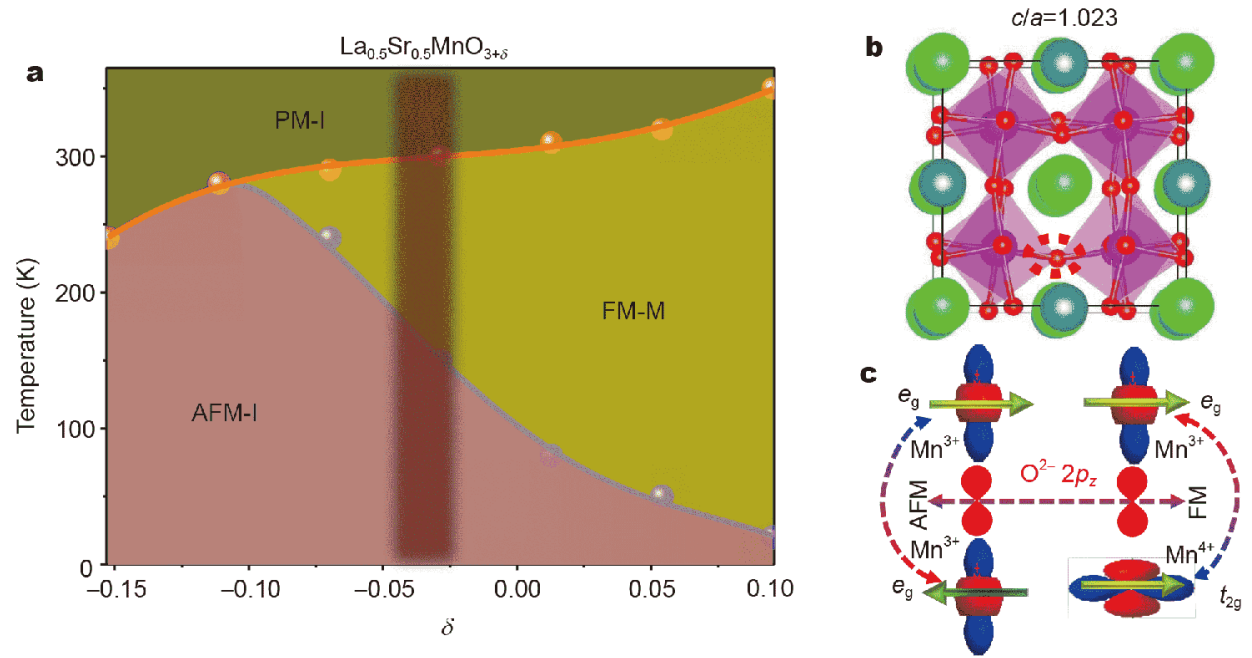

Figure 5 Temperature- $\delta$ phase diagram of $\mathrm{LSMO}_{3+\delta}$ and mechanism of phase transition. (a) Temperature-oxygen stoichiometry $(\delta)$ phase diagram in LSMO films. The electronic states (metal: M, insulating: I) are indicated as well as magnetic structures [paramagnetic: PM (dark yellow), ferromagnetic: FM (yellow), antiferromagnetic: AFM (orange)], red rectangular area indicates the hidden metal-insulator transition. (b) The perovskite structure of LSMO where the red dotted circle denotes the position of an oxygen vacancy. (c) Schematic illumination for the physical mechanism, showing that oxygen in the LSMO films has a significant influence on the Mn-O-Mn orbital hybridization through double exchange and superexchange.

1,073 K). Fig. 4b shows inhomogeneous FM ground states and due to large stray field from magnetic domains, AFM phase cannot be detected. As the magnetic field increases, MFM phase contrast gets stronger and $5 \mathrm{~T}$ is strong enough that it can align all the FM moments in the direction of applied magnetic field. In Fig. 4e (5 T) negative signal (blue) is from FM phase due to attractive force between tip and sample; positive signal (red) is from AFM phase due to repulsive force between tip and sample. Repulsive force at AFM region is due to the opposite stray field from FM region which is one of the direct evidences about AFM phase (see Fig. S5 for more details) in LSMO thin film grown at $T_{\text {atm }}=1,073 \mathrm{~K}$ using laser-ablated CVD system. Pure ferromagnetic $\mathrm{La}_{0.67} \mathrm{Sr}_{0.33} \mathrm{MnO}_{3} /$ $\mathrm{LaAlO}_{3}$ thin film (Fig. S6) is used for comparison which shows a saturated magnetic domain when it is exposed to magnetic field. MFM for the metallic thin film $\left(T_{\mathrm{atm}}=\right.$ $1,173 \mathrm{~K}$ ) by laser-ablated CVD (Fig. S7) also shows a much smaller areal ratio of AFM phase in local areas. The corresponding AFM and FM areas in LSMO films as a function of external field are fitted by exponent curves (Fig. S8), which reveals intrinsically the competition between AFM and FM phase modulated by the change of oxygen content.

The electronic and magnetic phase diagram as a function of $\delta$ is shown in Fig. 5a. Phase transformation from PM-I (I: insulating) phase to AFM-I phase occurs in samples with $\mathrm{V}_{\mathrm{O}}$ when temperature decreases. When the concentration of $\mathrm{V}_{\mathrm{O}}$ is reduced gradually, a hidden AFMI/FM-M (M: metallic) transition (red rectangular area) appears at low temperature. FM-M phase predominates in the samples with $\mathrm{E}_{\mathrm{O}}$. Fig. $5 \mathrm{~b}$ shows that when LSMO thin film suffers compressive strain $(\sim-1.7 \%)$ from LAO substrate and has a lattice distortion of $c / a=1.023$, oxygen octahedra is compressed in the plane and the films favor the C-type AFM-I ground states [26-28,39]. The magnetic property of LSMO, such as different oxidation states of $\mathrm{Mn}$ (i.e., $\mathrm{Mn}^{3+}$ and $\mathrm{Mn}^{4+}$ ), can be mediated by the double exchange mechanism as well as the superexchange mechanism (Fig. 5c). The interaction between $\mathrm{Mn}$ and $\mathrm{O}$ orbitals is essential for both of the two mechanisms. Apparently, $\mathrm{V}_{\mathrm{O}} / \mathrm{E}_{\mathrm{O}}$ can change the rotation and tilt of the oxygen octahedral $[40,41]$, which will undoubtedly influence the hybridization between $\mathrm{Mn} 3 \mathrm{~d}$ orbitals and $\mathrm{O} 2 \mathrm{p}$ orbitals via the variations of $\mathrm{Mn}-\mathrm{O}$ bond length and $\mathrm{Mn}-\mathrm{O}-\mathrm{Mn}$ bond angle. More importantly, one $\mathrm{V}_{\mathrm{O}}$ could offer two electrons and change the valence of $\mathrm{Mn}$ cations, meaning that some $\mathrm{Mn}^{4+} \mathrm{ca}-$ tions transform into $\mathrm{Mn}^{3+}$. As the simple picture illustrated in Fig. $5 c$, neighboring Mn cations with different valences $\left(\mathrm{Mn}^{3+}\right.$ and $\left.\mathrm{Mn}^{4+}\right)$ favor the FM configuration via double exchange interaction while $\mathrm{Mn}^{3+}$ cations neighboring to $\mathrm{Mn}^{3+}$ cations favor the AFM configuration via superexchange interaction. Therefore, a relatively high concentration of $\mathrm{V}_{\mathrm{O}}$ will stabilize the AFM state over FM state. Furthermore, the effect of oxygen stoichiometry on 
the electric property is reflected by oxygen influence on the concentration and mobility of the charge carriers. In the p-type LSMO films, holes are the majority carriers, and can recombine with the two electrons donated by every $\mathrm{V}_{\mathrm{O}}$. Such decreased carrier density leads to a low conductivity and the insulating behaviors in the films that contain rich $\mathrm{V}_{\mathrm{O}}$. On the contrary, $\mathrm{E}_{\mathrm{O}}$ increases the hole carrier concentration and improves the metallic conductivity. Besides, first-principles calculations (Fig. S9) show that removing enough oxygen ions from bulk LSMO in a certain way can reduce the density of state near the Fermi level, which is compatible well with the experimental results.

\section{CONCLUSION}

In conclusion, a new pathway is developed to synthesize the epitaxial oxide thin films combined with an in-situ chemical process, where the oxygen stoichiometry can be tuned in oxides. LSMO is used as a model system to study the in-situ effect of chemical reactivity on the oxygen stoichiometry in epitaxial thin films. It is found that the electric/magnetic properties are very sensitive to the microscopic oxygen configuration, and the competition between the AFM and FM phases induced by the oxygen content change enriches the phase diagram of LSMO. Our home-designed laser-ablated CVD system provides a new method to precisely modulate the properties in the materials that are sensitive to anion-stoichiometry, especially near the phase boundaries.

\section{Received 16 July 2018; accepted 23 August 2018; published online 10 September 2018}

1 Cava RJ, Batlogg $\mathrm{B}$, Chen $\mathrm{CH}$, et al. Oxygen stoichiometry, superconductivity and normal-state properties of $\mathrm{YBa}_{2} \mathrm{Cu}_{3} \mathrm{O}_{7-\delta}$. Nature, 1987, 329: 423-425

2 Biškup N, Salafranca J, Mehta V, et al. Insulating ferromagnetic $\mathrm{LaCoO}_{3-\delta}$ films: a phase induced by ordering of oxygen vacancies. Phys Rev Lett, 2014, 112: 087202

3 Skjærvø SH, Wefring ET, Nesdal SK, et al. Interstitial oxygen as a source of p-type conductivity in hexagonal manganites. Nat Commun, 2016, 7: 13745

4 Siemons W, Koster G, Yamamoto H, et al. Origin of charge density at $\mathrm{LaAlO}_{3}$ on $\mathrm{SrTiO}_{3}$ heterointerfaces: possibility of intrinsic doping. Phys Rev Lett, 2007, 98: 196802

5 Adler SB. Factors governing oxygen reduction in solid oxide fuel cell cathodes. Chem Rev, 2004, 104: 4791-4844

6 Yang CH, Seidel J, Kim SY, et al. Electric modulation of conduction in multiferroic Ca-doped $\mathrm{BiFeO}_{3}$ films. Nat Mater, 2009, 8: 485-493

7 Shu DJ, Ge ST, Wang M, et al. Interplay between external strain and oxygen vacancies on a rutile $\mathrm{TiO}_{2}(110)$ surface. Phys Rev Lett, 2008, 101: 116102

8 Kim YM, Morozovska A, Eliseev E, et al. Direct observation of ferroelectric field effect and vacancy-controlled screening at the $\mathrm{BiFeO}_{3} / \mathrm{La}_{x} \mathrm{Sr}_{1-x} \mathrm{MnO}_{3}$ interface. Nat Mater, 2014, 13: 1019-1025

$9 \mathrm{Ge} \mathrm{C}$, Jin $\mathrm{KJ}, \mathrm{Gu} \mathrm{L}$, et al. Metal-insulator transition induced by oxygen vacancies from electrochemical reaction in ionic liquidgated manganite films. Adv Mater Interfaces, 2015, 2: 1500407

10 Kourkoutis LF, Song JH, Hwang HY, et al. Microscopic origins for stabilizing room-temperature ferromagnetism in ultrathin manganite layers. Proc Natl Acad Sci USA, 2010, 107: 11682-11685

11 Li Z, Bosman M, Yang Z, et al. Interface and surface cation stoichiometry modified by oxygen vacancies in epitaxial manganite films. Adv Funct Mater, 2012, 22: 4312-4321

12 Muller DA, Kourkoutis LF, Murfitt M, et al. Atomic-scale chemical imaging of composition and bonding by aberration-corrected microscopy. Science, 2008, 319: 1073-1076

13 Nikolaenko YM, Artemov AN, Medvedev YV, et al. Non-uniform oxygen diffusion in epitaxial $\mathrm{La}_{0.7} \mathrm{Sr}_{0.3} \mathrm{MnO}_{3-\delta}$ film on $\mathrm{SrTiO}_{3}$ substrate. J Phys D-Appl Phys, 2016, 49: 375302

14 Lowndes DH, Geohegan DB, Puretzky AA, et al. Synthesis of novel thin-film materials by pulsed laser deposition. Science, 1996, 273: 898-903

15 Itina TE, Tokarev VN, Marine W, et al. Monte carlo simulation study of the effects of nonequilibrium chemical reactions during pulsed laser desorption. J Chem Phys, 1997, 106: 8905-8912

16 Geohegan DB. Imaging and blackbody emission spectra of particulates generated in the KrF-laser ablation of $\mathrm{BN}$ and $\mathrm{YBa}_{2} \mathrm{Cu}_{3} \mathrm{O}_{7-x}$. Appl Phys Lett, 1993, 62: 1463-1465

17 Lagrange JF, Hermann J, Wolfman J, et al. Time-resolved spatial distribution of plasma in the ablation of a $\mathrm{Ba}_{0.6} \mathrm{Sr}_{0.4} \mathrm{TiO}_{3}$ target by 25 ns KrF ultraviolet laser. J Appl Phys, 2014, 116: 133303

18 Lagrange JF, Hermann J, Wolfman J, et al. Dynamical plasma study during $\mathrm{CaCu}_{3} \mathrm{Ti}_{4} \mathrm{O}_{12}$ and $\mathrm{Ba}_{0.6} \mathrm{Sr}_{0.4} \mathrm{TiO}_{3}$ pulsed laser deposition by local thermodynamic equilibrium modelling. J Phys DAppl Phys, 2010, 43: 285202

19 Kim KS, Zhao Y, Jang H, et al. Large-scale pattern growth of graphene films for stretchable transparent electrodes. Nature, 2009, 457: 706-710

20 Tokura Y, Tomioka Y. Colossal magnetoresistive manganites. J Magn Magn Mater, 1999, 200: 1-23

21 Jin S, Tiefel TH, McCormack M, et al. Thousandfold change in resistivity in magnetoresistive La-Ca-Mn-O films. Science, 1994, 264: 413-415

22 Hemberger J, Krimmel A, Kurz T, et al. Structural, magnetic, and electrical properties of single-crystalline $\mathrm{La}_{1-x} \mathrm{Sr}_{x} \mathrm{MnO}_{3}(0.4<x<$ 0.85). Phys Rev B, 2002, 66: 094410

23 Moreo A. Phase separation scenario for manganese oxides and related materials. Science, 1999, 283: 2034-2040

24 Dagotto E, Hotta T, Moreo A. Colossal magnetoresistant materials: The key role of phase separation. Phys Rep, 2001, 344: 1-153

25 Gutiérrez D, Radaelli G, Sánchez F, et al. Bandwidth-limited control of orbital and magnetic orders in half-doped manganites by epitaxial strain. Phys Rev B, 2014, 89: 075107

26 Konishi Y, Fang Z, Izumi M, et al. Orbital-state-mediated phasecontrol of manganites. J Phys Soc Jpn, 1999, 68: 3790-3793

27 Pesquera D, Barla A, Wojcik M, et al. Strain-driven orbital and magnetic orders and phase separation in epitaxial half-doped manganite films for tunneling devices. Phys Rev Appl, 2016, 6: 034004

28 Radaelli G, Gutiérrez D, Qian M, et al. Strain-controlled responsiveness of slave half-doped manganite $\mathrm{La}_{0.5} \mathrm{Sr}_{0.5} \mathrm{MnO}_{3}$ layers inserted in $\mathrm{BaTiO}_{3}$ ferroelectric tunnel junctions. Adv Electron 
Mater, 2016, 2: 1600368

29 Cui B, Song C, Gehring GA, et al. Electrical manipulation of orbital occupancy and magnetic anisotropy in manganites. Adv Funct Mater, 2015, 25: 864-870

30 Vaz CAF, Moyer JA, Arena DA, et al. Magnetic and electronic structure of ultrathin $\mathrm{La}_{1-x} \mathrm{Sr}_{x} \mathrm{MnO}_{3}$ films at half doping. Phys Rev B, 2014, 90: 024414

31 van den Brink J, Khaliullin G, Khomskii D. Charge and orbital order in half-doped manganites. Phys Rev Lett, 1999, 83: 51185121

32 Peng JJ, Song C, Cui B, et al. Exchange bias in a single $\mathrm{LaMnO}_{3}$ film induced by vertical electronic phase separation. Phys Rev B, 2014, 89: 165129

33 Aruta C, Angeloni M, Balestrino G, et al. Preparation and characterization of lamno3 thin films grown by pulsed laser deposition. J Appl Phys, 2006, 100: 023910

34 Urushibara A, Moritomo Y, Arima T, et al. Insulator-metal transition and giant magnetoresistance in $\mathrm{La}_{1-x} \mathrm{Sr}_{x} \mathrm{MnO}_{3}$. Phys Rev B, 1995, 51: 14103-14109

35 Zhou H, Wang L, Hou Y, et al. Evolution and control of the phase competition morphology in a manganite film. Nat Commun, 2015, 6: 8980

36 Du K, Zhang K, Dong S, et al. Visualization of a ferromagnetic metallic edge state in manganite strips. Nat Commun, 2015, 6: 6179

37 Zhu Y, Du K, Niu J, et al. Chemical ordering suppresses large-scale electronic phase separation in doped manganites. Nat Commun, 2016, 7: 11260 manganites. Phys Rev Lett, 2000, 84: 3169-3172

39 Yi D, Flint CL, Balakrishnan PP, et al. Tuning perpendicular magnetic anisotropy by oxygen octahedral rotations in $\left(\mathrm{La}_{1-x} \mathrm{Sr}_{x} \mathrm{MnO}_{3}\right) /\left(\mathrm{SrIrO}_{3}\right)$ superlattices. Phys Rev Lett, 2017, 119: 077201

40 Liao Z, Huijben M, Zhong Z, et al. Controlled lateral anisotropy in correlated manganite heterostructures by interface-engineered oxygen octahedral coupling. Nat Mater, 2016, 15: 425-431

Acknowledgements This work was financially supported by the National Key Research and Development Program of China (2016YFA0302300). Zhang J also acknowledges the support from the National Natural Science Foundation of China (51332001) and the Fundamental Research Funds for the Central Universities (2017EYT26).

Author contributions Zhang J and Song C conceived the experiments and prepared the manuscript. Song $\mathrm{C}$ prepared the samples and performed the electronic transport, magnetic, XPS measurement. Malik IA performed the MFM measurement. Duan W and Li M performed the DFT calculation. Gu L and Zhang Q designed and performed the (S) TEM measurements. Wang L, Wang J, Chen R, Zheng R, Dong $S$ and Nan CW were involved in the revision of the manuscript. All authors were involved in the analysis of the experimental and theoretical results.

Conflict of interest The authors declare no conflict of interest.

Supplementary information Experimental details and supporting data are available in the online version of the paper. 


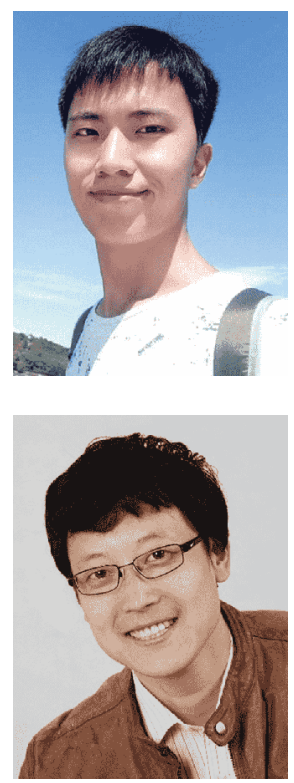

Chuangye Song obtained his Bachelor's degree in physics from Zhengzhou University in 2014. After that, he continued his education as a PhD candidate under the supervision of Prof. Jinxing Zhang at the Department of Physics, Beijing Normal University. His research interest now is the epitaxial growth of correlated-electron oxide film and the surface novel electronic phases at atomic scale by scanning tunneling microscopy in extreme conditions.

Jinxing Zhang obtained his PhD from The Hong Kong Polytechnic University in 2009 under the supervision of Prof. Helen Chan. After that he continued his research work at the Department of Physics of University of California, Berkeley as a post-doc scholar at Professor R. Ramesh's group. In 2012, he joined the Department of Physics, Beijing Normal University as a professor. The central goal of Zhang's group is the pursuit of the emerging phenomena and exotic physical behaviors behind the coupling and control of multiple order parameters (e.g., lattice, spin, orbital, charges) at a reduced dimension. His team is striving to create a bridge between those fundamentally scientific discoveries in functional nanosystems and future possible applications such as sensing, actuation, data storage, energy conversion, quantum manipulation, etc.

\section{可控氧化方法制备的锰氧化物中隐藏的金属-绝缘体转变研究}

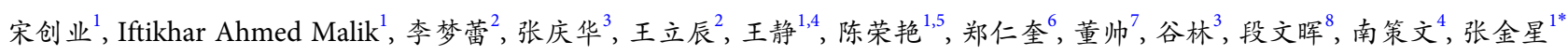

摘要 氧在调节复杂氧化物中的相结构和功能方面扮演着重要的角色, 比如高温超导体、庞磁电阻、催化等, 人们急需在这些功能氧化 物中实现有效且可控的氧含量调控. 在此工作中我们通过改变气体氛围温度来精确的调控氧化活性, 从而实现一种新的化学辅助的高质 量外延薄膜的制备方法. 以钙钛矿锰氧化物 $\mathrm{La}_{0.5} \mathrm{Sr}_{0.5} \mathrm{MnO}_{3}(\mathrm{LSMO})$ 为例, 通过调控薄膜样品中氧的化学计量比, 我们首次发现了该体系中 隐藏的由相竞争产生的金属-绝缘体转变. 氧组分引起的 Mn 离子间的交换相互作用以及载流子浓度的变化可能是此相转变产生的原因, 这与第一性原理计算十分符合. 不同于传统的阳离子掺杂, 有效的阴离子 $\left(\mathrm{O}^{2-}, \mathrm{S}^{2-}\right.$ 等 $)$ 调节提供了一种新的实现复杂氧化物外延薄膜中调 控相结构和功能的技术手段. 